\title{
Collateral Grades of the Willis' Circle Predicts Clinical Outcomes in Acute Intracranial Carotid Artery Occlusion before Thrombectomy
}

\author{
Hongchen Zhao MD; Qiang Dong MD PhD; Wenjie Cao MD PhD
}

Department of Neurology, Huashan Hospital, Fudan University, Shanghai, China

Objective Endovascular mechanical thrombectomy (EMT) promised acute ischemia stroke (AIS) with proximal artery occlusion significant but variable improvement of outcomes. We investigated the clinical outcome of EVMT in intracranial carotid artery occlusion (ICAO) and predictors of a favorable outcome.

\section{Methods Consecutive patients with acute ICAO who received EMT were}

retrospectively analyzed the pre-operative catheter angiographic finding. We derived a collateral grading system (primary collateral grade, $\mathrm{PCG}$ ) based on angiographic presentations, which categorized the collateral flow through Willis' circle into 3 grades: Grade I - anterior communicating artery (AcoA) was absent; Grade II - preserved AcoA with ipsilateral posterior communicating artery (iPcoA) absent; Grade III - both AcoA and iPcoA preserved (Figure 1). Logistic regression were used to assess the association of the PCG with the 90-day modified Rankin Scale (mRS) score.

Results The favorable clinical outcome at 90 days $(\mathrm{mRS} \leq 2)$ rated $31.6 \%(12 / 38)$.

The rate of successful reperfusion i.e. modified thrombolysis in cerebral infarction (mTICI) $\geq 2 \mathrm{~b}$ was $65.8 \%$ (25 in 38). PCG was associated with clinical outcomes as $61.5 \%$ patients with PCG3 achieved $\mathrm{mRS} \leq 2$ at 90 days, while it was $37.5 \%$ and $5.6 \%$ in patients with PCG-2 and PCG1(Table 1). PCG predicted $\mathrm{mRS} \leq 2$ at 90 days with an adjusted OR of 5.27, 95\% CI 1.31-21.13, $\mathrm{p}=0.019)$.

Conclusion PCG based on the integrity of Willis' Circle had strong power to predict prognosis of AIS patients with ICAO in EVMT, favorable functional outcome favored in patients with preserved both AcoA and ipsilateral PcoA.

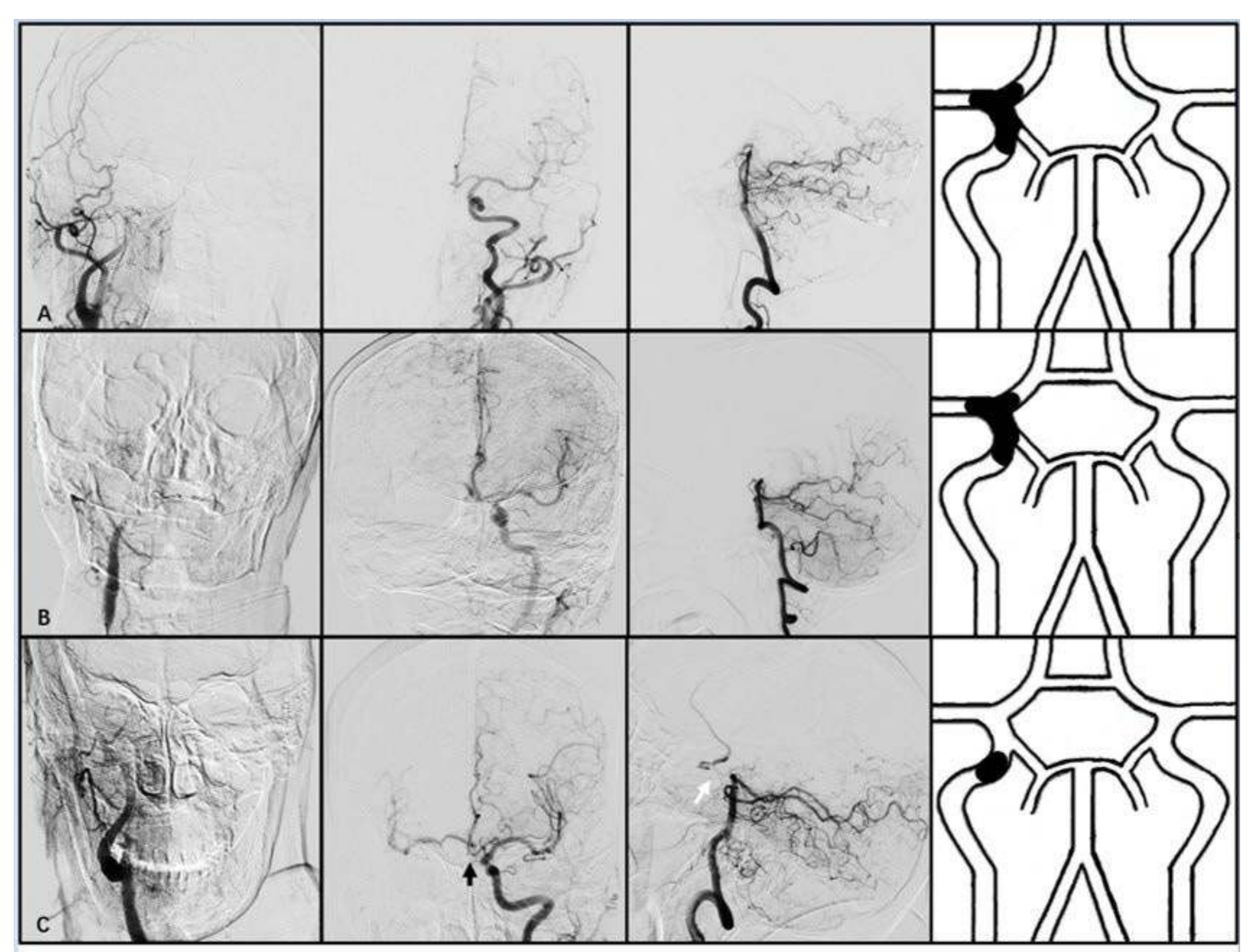

Figure 1. row A - PCG1, anterior communicating artery (AcOA) was absent, no matter ipsilateral posterior communicating artery (iPcoA) preserved or not; row B - PCG2, preserved AcoA with PCOA absent; row C - PCG3, both AcoA and iPcoA preserved. Black arrow - AcoA, white arrow - iPcoA.
Table 1. Univariate analysis between groups of different clinical outcomes

\begin{tabular}{lcccc}
\hline Clinical outcome & $\begin{array}{c}\text { Total } \\
(\mathbf{n}=\mathbf{3 8})\end{array}$ & $\begin{array}{c}\text { Favorable } \\
(\mathbf{n}=\mathbf{1 2})\end{array}$ & $\begin{array}{c}\text { Unfavorable } \\
(\mathbf{n}=\mathbf{2 6})\end{array}$ & $\begin{array}{l}\mathbf{p} \\
\text { value }\end{array}$ \\
\hline Male, (\%) & $21(53.30)$ & $8(66.67)$ & $13(50.00)$ & 0.337 \\
Age (yrs), median & $77.5(69.0-81.0)$ & $68.5(50.5-76.75)$ & $79.5(74-82)$ & 0.015 \\
Hypertension (\%) & $25(65.80)$ & $6(50.00)$ & $19(73.08)$ & 0.163 \\
Diabetes (\%) & $5(13.20)$ & $0(0.00)$ & $5(19.23)$ & 0.103 \\
Atrial Fibrillation & $22(57.90)$ & $8(66.67)$ & $14(53.85)$ & 0.457 \\
(\%) & & & & \\
Admission NIHSS & $23.0(18.0-29.25)$ & $18(15-22.50)$ & $25.5(21.75-31.75)$ & 0.001 \\
ASPECT & $7.5(6.0-9.0)$ & $8(6-9)$ & $7(6-8.25)$ & 0.466 \\
BG, mean \pm SD & $7.97 \pm 2.27$ & $7.28 \pm 2.01$ & $8.29 \pm 2.34$ & 0.206 \\
SBP, mean \pm SD & $154.37 \pm 29.53$ & $148.83 \pm 22.79$ & $156.92 \pm 32.25$ & 0.440 \\
DBP, mean \pm SD & $83.45 \pm 16.26$ & $83.00 \pm 18.62$ & $83.65 \pm 15.45$ & 0.910 \\
IV-tPA (\%) & $16(42.10)$ & $6(50.00)$ & $10(38.46)$ & 0.503 \\
OTP, mins, median & $345(236.75-391)$ & $326(213-451)$ & $349(245-388.50)$ & 0.816
\end{tabular}

Primary collateral grade $(\%)$

$\begin{array}{lcccc}\text { I } & 17(44.70) & 1(8.33) & 16(61.54) & 0.005 \\ \text { II } & 8(21.10) & 3(25.00) & 5(19.23) & \\ \text { III } & 13(34.20) & 8(66.67) & 5(19.23) & \\ & & 11(91.67) & 14(53.85) & 0.022\end{array}$

